10.21611/qirt.2019.048

\title{
Simultaneous Determination of Emissivity and Temperature of Titanium at High Temperature
}

\author{
by Sumeet Kumar*, C. V. Krishnamurthy*, Krishnan Balasubramaniam** \\ * Department of Physics, Indian Institute of Technology Madras, Chennai 600036, India, email: \\ sumeetk@physics.iitm.ac.in \\ ${ }_{* *}$ Department of Mechanical Engineering, Indian Institute of Technology Madras, Chennai 600036, India
}

\begin{abstract}
Multispectral radiation thermometry (MRT) has been implemented to estimate the temperature of roughened titanium surface at 693 and $843 \mathrm{~K}$. The radiance is measured at four wavelengths, 1.625, 2.3, 5.14 and $7.8 \mu \mathrm{m}$, and is fitted to an emissivity model having one or two unknown coefficients. Analytical and empirical models, thirteen in all, are examined for suitability under typical conditions of surface roughness, high temperature and different heating times. No single model is found to capture all the conditions. However, two analytical models are found to account for surface roughness and provide good estimates of temperature when oxide layer formation is not significant.
\end{abstract}

\section{Introduction}

Titanium alloys are used for high temperature applications in gas turbine industries [1]. Measurement of temperature from thermocouple is not viable in such an environment. Noncontact technique like radiation thermometry can be implemented to get a good estimate of temperature. The most common approach is spectral radiation thermometry (SRT) in which temperature is evaluated from known emissivity or vice versa from the radiance measured at a wavelength. The accuracy of input emissivity is very important as even a small error could result into a large difference in estimated temperature from the true temperature. SRT technique has been implemented by Zhang et al. [2], $\mathrm{Li}$ et al. [3] and others to determine the emissivity of pure titanium and titanium alloys. Zhang et al. evaluated the emissivity of pure titanium in a spectral range of $3-27 \mu \mathrm{m}$ while heating the roughened sample surface at $873 \mathrm{~K}$ in air for $24 \mathrm{~h}$. Similarly Li et al. heated Ti-6Al-4V alloy at $923 \mathrm{~K}$ in air and determined the emissivity of the alloy in the spectral range of $1.5-2.2 \mu \mathrm{m}$. It has been found that small variation in parameters like wavelength, temperature, heating time, surface roughness or impurities cause a significant change in emissivity [4,5]. Due to such complexities, it is better to use the published emissivity data only in case of similar experimental conditions for determining the temperature from SRT.

Multispectral radiation thermometry (MRT) is another technique by which temperature can be estimated even from unknown emissivity. The measured radiance at $n$ or more wavelengths are fitted to an emissivity model having $n-2$ unknown coefficients. Wen et al. [6,7] reported about the optimized value of $n$ for polished and roughened aluminum alloy surfaces. They heated both types of samples at 600 and $700 \mathrm{~K}$ and estimated the temperature from MRT using the data obtained for $n, n+1$ and $n+2$ wavelengths. No significant improvements in estimated temperatures were found due to the involvement of more number of wavelengths for measurements.

In the present work, an experimental setup is fabricated to estimate the temperature of titanium samples from MRT using radiance measured at wavelengths of 1.625, 2.3, 5.14 and $7.8 \mu \mathrm{m}$. The parameters like temperature, surface roughness and heating time are varied and temperature is estimated under these conditions from both analytical and empirical emissivity models. The absolute temperature differences between true and estimated temperatures are calculated for analyzing the results. Finally the models which would be able to predict emissivity of titanium under various conditions are suggested.

\section{Measurement methods}

The radiation emitted by an object depends on its emissivity. The emissivity of a blackbody is 1 at all wavelengths in the entire hemispherical region. That is why it emits the most among all the objects at the same temperature. For a real object at temperature $T$, emissivity is the ratio of radiation intensity emitted by the object and a blackbody at the same temperature. The radiance of a blackbody is expressed as Planck's function. In certain algorithms like SRT, Wien's formula is used instead of Planck's function for near and mid infrared regions because of its simpler mathematical form. The following expression is used in SRT to determine the temperature of an object from the measured radiance for a given emissivity at a specified wavelength.

$$
\frac{1}{T}=\frac{1}{T_{b}}+\frac{\lambda}{C_{2}} \log \epsilon
$$

where $T$ is the temperature of the object, $T_{b}$ is the temperature of a blackbody emitting the radiation equivalent to the radiation leaving the object surface at temperature $T, \lambda$ is the wavelength, $C_{2}$ is a constant equals to $0.014388 \mathrm{~m} \mathrm{~K}$ and $\epsilon$ 
is the emissivity of the object. In Eq. (1), the effect of surrounding in the radiation leaving the surface of the object has been ignored. This is a valid assumption for objects at high temperature [8].

The temperature of the object can be determined from MRT even for an unknown emissivity. In this technique, radiance is measured at three or more wavelengths. The measured data is fitted to a suitable emissivity model which is capable of describing the emissivity variation at least within the range of operating wavelengths. Least squares method is implemented further to get the best fit for the data with the model. The sum of the squares of the differences of $\left(1 / T_{b}\right)$ obtained from experiment and model at various wavelengths is minimized in this method. The quantity to be minimized is termed as $x^{2}$ which is given by

$$
\chi^{2}=\sum_{i=1}^{N}\left(\frac{1}{T_{b, i}^{e x p}}-\frac{1}{T_{b, i}^{\text {model }}}\right)^{2}
$$

where $N$ is the number of wavelengths, $T_{b, i}^{e x p}$ and $T_{b, i}^{\text {model }}$ are the values of $T_{b}$ obtained from experiment and model respectively at wavelength $\lambda_{i}$. In this way, the unknown coefficients present in the model and temperature of the object are determined from the best fit to the model. The choice of emissivity model is very crucial in MRT for making an estimation of temperature closer to the true temperature of the object. There are few emissivity models like Hagen Rubens (HR) model [4] and Agababov model $[9,10]$ which are derived analytically under certain assumptions. Several empirical models [6] are also available for estimating the temperature from MRT. These empirical models include polynomial, logarithmic or exponential expressions of emissivity as a function of wavelength and/or temperature.

\subsection{Hagen - Rubens relation}

When an electromagnetic wave is incident on a smooth surface of an opaque object at an angle of incidence $\theta$, it gets reflected and refracted from the air - object interface. The reflectance $R$ of the object surface for the wave can be calculated from Fresnel equations. Kirchhoff's law is used to relate the reflectance and emissivity of the object by an equation given as $\epsilon=1-R$. For normal incident wave, the emissivity relation is reduced to a simpler mathematical form.

$$
\epsilon=\frac{4 n}{(n+1)^{2}+\kappa^{2}}
$$

where $n$ and $k$ are the refractive index and extinction coefficient of the object respectively. For long wavelengths $(\lambda>\sim 5$ $\mu \mathrm{m})$ and for smaller resistivity $r_{e}$ materials like metals, Eq. (3) is simplified further such that the emissivity depends on only one variable. On considering only dominant term present in the expansion of Eq. (3), final form of the emissivity relation is expressed as

$$
\epsilon=36.5 \sqrt{\left(r_{e} / \lambda\right)}
$$

where $r_{e}$ and $\lambda$ are in $\Omega \mathrm{cm}$ and $\mu \mathrm{m}$ respectively. Eq. (4) is known as Hagen - Rubens relation.

\subsection{Agababov model}

Agababov proposed a model to relate the emissivity of an object having rough and smooth surfaces using geometric optics approximation. The derivation has been done for an inverted conical like structure assuming that the inner surface of the cone represents rough surface and the circular base would have represented the smooth surface. The surfaces are assumed to be having thermal and optical homogeneity.

$$
\epsilon_{r}=\left(1+\left(\frac{1}{\epsilon_{s}}-1\right) R\right)^{-1}
$$

where $\epsilon_{r}$ and $\epsilon_{s}$ are the effective emissivity of the rough and smooth surfaces respectively and $R$ is the ratio of the areas of circular base and surface area of the cone i.e. $R=A_{s} / A_{r}$. In another work [11], Agababov related the quantity $R$ for a random isotropic rough surface with its characterized roughness parameters experimentally. The roughness parameters include $n$ and $\sigma$ which indicate the number of intersections of the surface profile with the mean per unit length of the mean line and root mean square of the surface profile from the mean respectively. It was found that $R$ is equivalent to the following term.

$$
R=\left(1+\pi^{2} n^{2} \sigma^{2}\right)^{-1}
$$

Hagen - Rubens relation contains a term $r_{e}$ as given in Eq. (4) which is a function of temperature $T$. Before using the relation in MRT for fitting, it has been modified such that the model would contain two independent unknown quantities $a_{0}$ and $T$. In Agababov model, the quantity $R$ is constant for a surface as given in Eq. (6). Thus it can be considered as another unknown coefficient $a_{1}$ present in the emissivity model for MRT. In the present work, HR model 


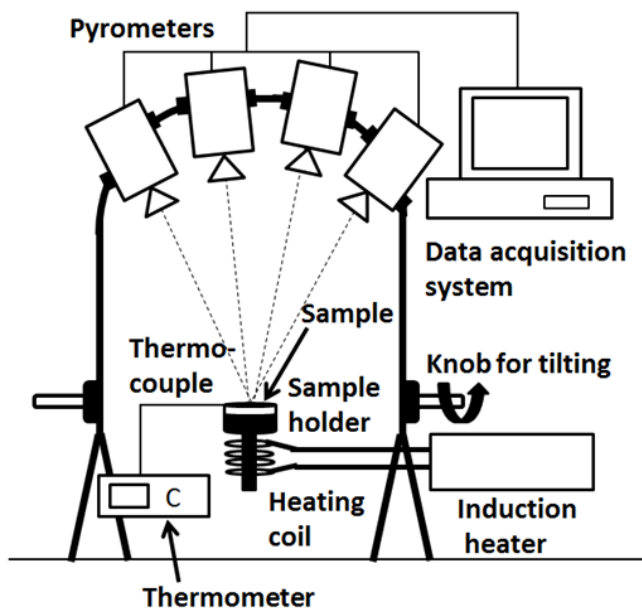

(a)
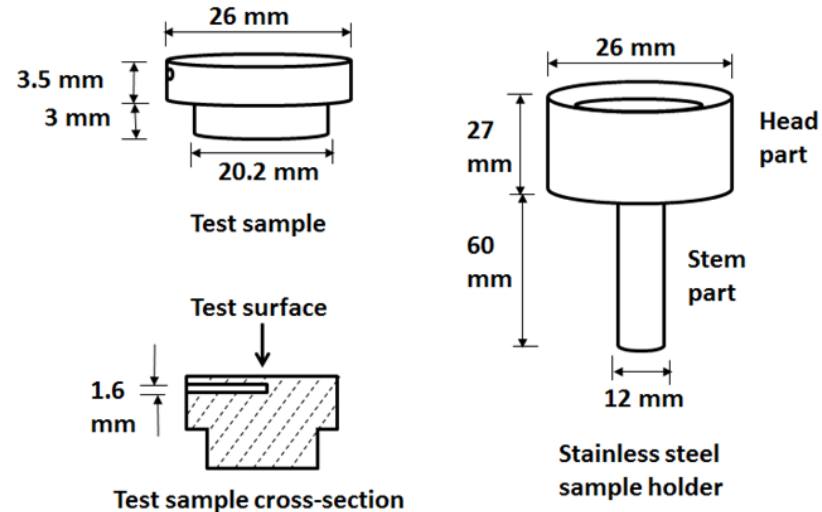

(b)

Fig. 1: Schematic diagram of the (a) emissivity measurement experimental setup; and (b) sample and sample holder.

and combination of HR and Agababov models (HR,A) are implemented as emissivity models for fitting the experimental data. The mathematical forms of both the models are given in table 1.

\section{Experimental setup and procedure}

An experimental setup is fabricated for determining the temperature of metals from MRT at industrial sites as shown in figure 1. The heating and measurement units are two main components of the setup. The heating unit contains a K-type thermocouple, stainless steel sample holder, heating coil and induction heating controlling unit. A disc shaped metallic sample is placed over the head part of the sample holder. The stem part of the sample holder is passed through the axis of a helical shaped heating coil. In this way, the sample holder is firstly heated from induction technique which in turn heats up the sample through thermal conduction. A hole of diameter $1.6 \mathrm{~mm}$ is drilled just below the top surface of the sample for measuring the true temperature at the centre of the sample. Water is continuously flowed through the coil for maintaining its temperature near ambient. The temperature of the sample can be changed by varying the current passing through the coil. The radiation leaving the sample surface is measured by four narrow bandwidth IMPAC pyrometers operating at wavelengths of $1.625,2.3,5.14$ and $7.8 \mu \mathrm{m}$. The pyrometers are slightly tilted $\left(<15^{\circ}\right)$ such that all of them can collect radiation from the same region. The measured radiance is converted into temperature $T$ as given in Eq. (1). The involvement of less optical and electronic components in the setup makes it feasible to easily move and fix at various industrial sites.

The samples are prepared by polishing the pure titanium surfaces from SiC abrasive sheets and diamond paste. Three different roughness are given to the sample surfaces and are labelled as sample 1, 2 and 3 . Sample 1 is given mirror finish by polishing the surface from a series of $\mathrm{SiC}$ abrasive sheets of different grit sizes ranging from 320 to 1500 and diamond paste. The roughness is produced to the surface of sample 2 by polishing with 320, 400, 600 and 800 grit sizes SiC abrasive sheets. Sample 3 is prepared by bombarding the surface with alumina using sandblasting technique. Two sets of samples 1, 2 and 3 are fabricated for heating at two different temperatures. All the samples are cleaned with ethanol and acetone for removing any sort of dust, grease or oil. The surface roughnesses of the samples are measured by surface profilometer and the values of $\sigma$ for samples 1, 2 and 3 are 96, 550 and $1500 \mathrm{~nm}$ respectively. Samples in set 1 and set 2 are heated in air at 693 and $843 \mathrm{~K}$ respectively. The radiance is measured after heating the samples for $1,5,12,35,65,105,145,190,240,280$ and 325 min from all the pyrometers.

\section{Results and discussion}

Emissivity of the samples are determined by SRT from Eq. (1) at 693 and $843 \mathrm{~K}$ at eleven different heating times in between 1 and $325 \mathrm{~min}$ as shown in table 2. The effects of three important parameters i.e. temperature, surface roughness and heating time on emissivity are analysed. Emissivity of samples 1,2 and 3 are increasing with heating time at $693 \mathrm{~K}$ due to the formation of oxide layer. But at $843 \mathrm{~K}$, a maxima in emissivity has been observed after heating the samples for certain period of time at wavelengths of 1.625 and $2.3 \mu \mathrm{m}$. For example, the emissivity of sample 1 has increased to a maximum value of 0.89 at wavelength of $1.625 \mu \mathrm{m}$ after heating for $65 \mathrm{~min}$ at $843 \mathrm{~K}$. Unlike the samples at $693 \mathrm{~K}$, a non-monotonic behaviour in emissivity versus wavelength is found for samples heated at $843 \mathrm{~K}$ for different heating times. This effect is prominent for sample 1 in comparison to samples 2 and 3.

The temperatures of samples are estimated from MRT by fitting the experimental data to the emissivity models as given in tables 3 and 4 . The effectiveness of the models are quantified by calculating the absolute temperature difference $\Delta T$ between the true and estimated temperatures. Other statistical quantities like $R^{2}$ and standard error in 
estimated temperature are used to analyse the results. Firstly the analytical models HR and (HR,A) are implemented for samples at 693 and $843 \mathrm{~K}$. For mirror surface finish i.e. sample 1, the values of $\Delta T$ from HR are even less than $2 \mathrm{~K}$ on heating the sample at $693 \mathrm{~K}$ for different heating times. The standard errors in temperatures are lying in between 4 and 7 $\mathrm{K}$ and the values of $R^{2}$ are above 0.99 . Overall a good estimation of temperature is made by MRT for sample 1 at $693 \mathrm{~K}$ from HR model. But the values of $\Delta T$ from HR are nearly 20 and $30 \mathrm{~K}$ for samples 2 and 3 respectively heated at the same temperature which are larger compared to sample 1. (HR,A) model is implemented further on the same experimental data. The estimated temperatures have approached closer to the true temperature from $(\mathrm{HR}, \mathrm{A})$ as the values of $\Delta T$ have reduced to less than 3 and $4 \mathrm{~K}$ for samples 2 and 3 respectively. The standard errors in temperatures are exhibiting from 12 to $15 \mathrm{~K}$ for sample 2 whereas for sample 3, the values are lying in between 4 and $8 \mathrm{~K}$. The values of $R^{2}$ are more than 0.99 for both the samples from (HR,A). An oxide layer of certain thickness has formed on heating the samples at $693 \mathrm{~K}$. From the results, it seems like the models which are derived for bare surface metals i.e. HR and (HR,A) are still applicable. This means that the effect of surface properties of substrate is dominant over the film because of its smaller thickness.

The samples 1, 2 and 3 from another set are heated at $843 \mathrm{~K}$ and the results are analysed in the similar way like previous case as shown in table 3 . The values of $\Delta T$ from HR are very large for sample 1 . In fact the value has reached to $71.34 \mathrm{~K}$ for the sample heated for $145 \mathrm{~min}$. HR and $(\mathrm{HR}, \mathrm{A})$ are fitted to data obtained for samples 2 and 3 at $843 \mathrm{~K}$. Better temperature estimations are obtained from HR than (HR,A) for most of the cases. But the values of $\Delta T$ are quite large of nearly 15 and $30 \mathrm{~K}$ for samples 2 and 3 respectively from HR. A thicker oxide layer has formed on the surfaces of samples heated at $843 \mathrm{~K}$ than that for samples heated at $693 \mathrm{~K}$. Unlike the previous case i.e. $693 \mathrm{~K}$, the effect of oxidation is dominant over the substrate surface properties. That is why the analytical models derived for bare metal surfaces could not estimate the temperatures of samples for most of the cases. The estimated temperatures for samples 2 and 3 from HR are closer to true temperature in comparison with the estimated temperatures for sample 1 from the same model. This conveys that the combination of surface roughness and oxide layer has produced better result than polished oxidized surface at $843 \mathrm{~K}$.

A set of eleven existing empirical models are fitted further for finding the behaviour of emissivity of samples heated at $843 \mathrm{~K}$ for different heating times. The mathematical expressions of empirical models labelled as EM1 to EM11 are given in table 1 . The models include polynomial and exponential functions of emissivity with wavelength and/or temperature having one or two unknown coefficients. The values of $\Delta T$ obtained from fitting are given in table 4 . Models EM6 and EM8 have made better estimation of temperatures for shorter heating times (1 to 65 min) whereas for longer heating times (105 to $325 \mathrm{~min}$ ), models EM1 and EM3 have estimated the temperatures closer to the true temperature. Overall it is difficult to choose a particular model which can estimate temperature at different heating times for all the samples. The models which have fitted well to the experimental data are shortlisted as shown in table 5 . The standard errors in estimated temperatures and the values of $R^{2}$ for the best fitted models are also shown in the table. The values of $R^{2}$ are above 0.96 which implies that a good fitting is done with the experimental data. But the standard errors in estimated temperatures are quite large for most of the cases which explain about the scattered estimated temperature values from the measured values at different wavelengths. In this case, it is difficult to describe the emissivity behaviour by a particular model.

\section{Conclusion}

The temperature of roughened oxidized titanium samples are estimated using MRT by fitting to both analytical and empirical emissivity models. A good estimation of temperature is made from $\mathrm{HR}$ and combination of HR and Agabobov models for polished and roughened surfaces respectively at $693 \mathrm{~K}$. But at $843 \mathrm{~K}$, the temperatures estimated from these analytical models are not very close to the true temperature. Formation of thicker oxide layer has taken place for samples at $843 \mathrm{~K}$ than $693 \mathrm{~K}$. The analytical models can be used to estimate the temperatures of samples as long as the oxide layer has been barely present on the substrate surface. An attempt has been made to describe the spectral behavior of emissivity of roughened titanium surface at $843 \mathrm{~K}$ during the heating process. The radiance data is fitted to eleven existing empirical models. But there is no single model which can make a good estimation of temperature at 893 $\mathrm{K}$ under all these conditions.

\section{REFERENCES}

[1] Winstone M, Strang A. Titanium alloys at elevated temperature: structural development and service behaviour. London: Maney Publishing, 2001.

[2] Zhang F, Yu K, Zhang K, Liu Y, Zhao Y, Liu Y. Infrared spectral emissivity property of pure titanium in the 473$1035 \mathrm{~K}$ temperature range. Appl. Spectrosc. 2016;70(10):1717-1725.

[3] Li L, Yu K, Zhang K, Liu Y. Study of Ti-6Al-4V alloy spectral emissivity characteristics during thermal oxidation process. Int. J. Heat Mass Transfer 2016;101:699-706.

[4] Siegel R, Howell JR. Thermal radiation heat transfer. New York: Taylor \& Francis, 2002.

[5] Modest MR. Radiative heat transfer. New York: McGraw-Hill International Editions, 1993.

[6] Wen C, Mudawar I. Emissivity characteristics of polished aluminum alloy surfaces and assessment of multispectral radiation thermometry (MRT) emissivity models. Int. J. Heat Mass Transfer 2005;48:1316-1329.

[7] Wen C, Mudawar I. Emissivity characteristics of roughened aluminium alloy surfaces and assessment of multispectral radiation thermometry (MRT) emissivity models. Int. J. Heat Mass Transfer 2004;47:3591-3605. 
[8] Perez-Saez RB, del Campo L, Tello MJ. Analysis of the accuracy of methods for the direct measurement of emissivity. Int. J. Thermophys 2008;29:1141-1155.

[9] Agababov SG. Effect of the roughness of the surface of a solid body on its radiation properties and methods for their exponential determination, Teplofizika Vyokikh Temperature 1968;6:78-87.

[10] Wen C, Mudawar I. Modeling the effects of surface roughness on the emissivity of aluminium alloys. Int. J. Heat Mass Transfer 2006;49:4279-4289.

[11] Agababov SG. Effect of secondary roughness on the emissive properties of solid bodies. Teplofizika Vysokikh Temperature 1970;8:220-222.

Table 1. Mathematical form of emissivity models examined in this study.

\begin{tabular}{ccl|cll}
\hline SI. No. & Model & Expression & SI. No. & Model & Expression \\
\hline 1 & HR & $\epsilon=a_{0}(T / \lambda)^{1 / 2}$ & 8 & EM6 & $\epsilon=\exp \left(a_{0} / T_{b}\right)$ \\
2 & $(\mathrm{HR}, \mathrm{A})$ & $\epsilon=\left(1+\left(a_{0}^{-1}(T / \lambda)^{-1 / 2}-1\right) a_{1}\right)^{-1}$ & 9 & EM7 & $\epsilon=\exp \left(a_{0}+a_{1} / T_{b}\right)$ \\
3 & EM1 & $\epsilon=\exp \left(a_{0}+a_{1} \lambda\right)$ & 10 & EM8 & $\epsilon=\exp \left(a_{0} \lambda^{1 / 2}\right)$ \\
4 & EM2 & $\epsilon=a_{0}+a_{1} \lambda$ & 11 & EM9 & $\epsilon=\exp \left(a_{0}+a_{1} \lambda^{1 / 2}\right)$ \\
5 & EM3 & $\epsilon=1 /\left(1+a_{0} \lambda^{2}\right)$ & 12 & EM10 & $\epsilon=\exp \left(a_{0} / \lambda^{1 / 2}\right)$ \\
6 & EM4 & $\epsilon=\exp \left(a_{0} \lambda+a_{1} T\right)$ & & EM11 & $\epsilon=\exp \left(a_{0}+a_{1} / \lambda^{1 / 2}\right)$ \\
7 & EM5 & $\epsilon=\exp \left(a_{0} \lambda+a_{1} / T_{b}\right)$ & & & \\
\hline
\end{tabular}

Table 2. Emissivity of samples evaluated from SRT technique.

\begin{tabular}{|c|c|c|c|c|c|c|c|c|c|c|c|c|c|}
\hline \multirow{3}{*}{$\begin{array}{l}\text { Tem- } \\
\mathrm{p}(\mathrm{K})\end{array}$} & \multirow{3}{*}{$\begin{array}{l}\text { Sa- } \\
\text { mple }\end{array}$} & \multirow{3}{*}{$\begin{array}{l}\text { Wave- } \\
\text { length } \\
(\mu \mathrm{m})\end{array}$} & \multicolumn{11}{|c|}{ Emissivity } \\
\hline & & & \multicolumn{11}{|c|}{ Heating time (min) } \\
\hline & & & 1 & 5 & 12 & 35 & 65 & 105 & 145 & 190 & 240 & 280 & 325 \\
\hline \multirow{12}{*}{693} & \multirow{4}{*}{1} & 1.625 & 0.47 & 0.47 & 0.47 & 0.48 & 0.48 & 0.49 & 0.49 & 0.50 & 0.51 & 0.51 & 0.52 \\
\hline & & 2.3 & 0.42 & 0.42 & 0.43 & 0.43 & 0.43 & 0.44 & 0.44 & 0.45 & 0.45 & 0.46 & 0.46 \\
\hline & & 5.14 & 0.26 & 0.26 & 0.26 & 0.27 & 0.27 & 0.27 & 0.27 & 0.28 & 0.28 & 0.28 & 0.28 \\
\hline & & 7.8 & 0.22 & 0.22 & 0.22 & 0.23 & 0.23 & 0.23 & 0.24 & 0.24 & 0.24 & 0.24 & 0.24 \\
\hline & \multirow{4}{*}{2} & 1.625 & 0.62 & 0.62 & 0.63 & 0.63 & 0.64 & 0.64 & 0.65 & 0.65 & 0.65 & 0.66 & 0.66 \\
\hline & & 2.3 & 0.59 & 0.60 & 0.60 & 0.60 & 0.61 & 0.61 & 0.62 & 0.62 & 0.62 & 0.62 & 0.62 \\
\hline & & 5.14 & 0.44 & 0.44 & 0.44 & 0.45 & 0.45 & 0.45 & 0.46 & 0.46 & 0.46 & 0.46 & 0.46 \\
\hline & & 7.8 & 0.38 & 0.38 & 0.38 & 0.39 & 0.39 & 0.39 & 0.39 & 0.40 & 0.40 & 0.40 & 0.40 \\
\hline & \multirow{4}{*}{3} & 1.625 & 0.69 & 0.69 & 0.69 & 0.70 & 0.71 & 0.71 & 0.72 & 0.72 & 0.73 & 0.73 & 0.74 \\
\hline & & 2.3 & 0.67 & 0.67 & 0.67 & 0.68 & 0.69 & 0.69 & 0.69 & 0.69 & 0.70 & 0.70 & 0.71 \\
\hline & & 5.14 & 0.54 & 0.54 & 0.55 & 0.56 & 0.56 & 0.57 & 0.57 & 0.57 & 0.57 & 0.57 & 0.58 \\
\hline & & 7.8 & 0.49 & 0.49 & 0.49 & 0.50 & 0.50 & 0.50 & 0.51 & 0.51 & 0.51 & 0.51 & 0.51 \\
\hline \multirow{12}{*}{843} & \multirow{4}{*}{1} & 1.625 & 0.61 & 0.63 & 0.67 & 0.80 & 0.89 & 0.88 & 0.82 & 0.76 & 0.68 & 0.64 & 0.64 \\
\hline & & 2.3 & 0.49 & 0.51 & 0.53 & 0.60 & 0.72 & 0.82 & 0.88 & 0.89 & 0.85 & 0.82 & 0.80 \\
\hline & & 5.14 & 0.28 & 0.29 & 0.29 & 0.30 & 0.31 & 0.33 & 0.35 & 0.36 & 0.38 & 0.40 & 0.42 \\
\hline & & 7.8 & 0.24 & 0.24 & 0.24 & 0.24 & 0.25 & 0.25 & 0.26 & 0.26 & 0.26 & 0.27 & 0.27 \\
\hline & \multirow{4}{*}{2} & 1.625 & 0.65 & 0.66 & 0.68 & 0.71 & 0.77 & 0.81 & 0.83 & 0.83 & 0.82 & 0.81 & 0.80 \\
\hline & & 2.3 & 0.59 & 0.60 & 0.61 & 0.64 & 0.68 & 0.72 & 0.76 & 0.79 & 0.81 & 0.82 & 0.83 \\
\hline & & 5.14 & 0.40 & 0.40 & 0.40 & 0.41 & 0.43 & 0.44 & 0.46 & 0.47 & 0.48 & 0.49 & 0.50 \\
\hline & & 7.8 & 0.34 & 0.34 & 0.34 & 0.34 & 0.35 & 0.36 & 0.36 & 0.37 & 0.37 & 0.37 & 0.38 \\
\hline & \multirow{4}{*}{3} & 1.625 & 0.84 & 0.85 & 0.86 & 0.87 & 0.87 & 0.87 & 0.86 & 0.86 & 0.85 & 0.85 & 0.85 \\
\hline & & 2.3 & 0.79 & 0.80 & 0.81 & 0.83 & 0.85 & 0.85 & 0.85 & 0.86 & 0.86 & 0.86 & 0.86 \\
\hline & & 5.14 & 0.61 & 0.61 & 0.62 & 0.63 & 0.65 & 0.66 & 0.67 & 0.68 & 0.69 & 0.69 & 0.70 \\
\hline & & 7.8 & 0.52 & 0.52 & 0.52 & 0.53 & 0.53 & 0.54 & 0.54 & 0.55 & 0.55 & 0.55 & 0.56 \\
\hline
\end{tabular}


Table 3. Absolute error in the estimated temperatures from $H R$ and $(H R, A)$ models.

\begin{tabular}{|c|c|c|c|c|c|c|c|c|c|c|}
\hline \multirow{4}{*}{$\begin{array}{l}\text { Heating } \\
\text { time } \\
\text { (min) }\end{array}$} & \multicolumn{10}{|c|}{$\Delta T=\mid T_{\text {True }}-T_{\text {Estimated }}(\mathrm{K})$} \\
\hline & \multicolumn{5}{|c|}{ Temperature $=693 \mathrm{~K}$} & \multicolumn{5}{|c|}{ Temperature $=843 \mathrm{~K}$} \\
\hline & \multirow{2}{*}{$\begin{array}{c}\text { Sam } 1 \\
\text { HR }\end{array}$} & \multicolumn{2}{|c|}{ Sample 2} & \multicolumn{2}{|c|}{ Sample 3} & \multirow{2}{*}{$\begin{array}{c}\text { Sam } 1 \\
\text { HR }\end{array}$} & \multicolumn{2}{|c|}{ Sample 2} & \multicolumn{2}{|c|}{ Sample 3} \\
\hline & & HR & $(\mathrm{HR}, \mathrm{A})$ & HR & $(\mathrm{HR}, \mathrm{A})$ & & HR & $(\mathrm{HR}, \mathrm{A})$ & HR & $(\mathrm{HR}, \mathrm{A})$ \\
\hline 1 & 0.42 & 19.59 & 1.27 & 30.75 & 0.18 & 15.19 & 12.17 & 23.96 & 30.50 & 16.05 \\
\hline 5 & 1.25 & 19.25 & 1.20 & 30.83 & 0.41 & 17.69 & 10.50 & 23.95 & 30.05 & 21.15 \\
\hline 12 & 1.55 & 19.05 & 0.86 & 30.63 & 2.82 & 23.75 & 8.95 & 23.65 & 28.25 & 33.29 \\
\hline 35 & 1.05 & 19.21 & 2.50 & 30.05 & 1.77 & 44.42 & 2.79 & 20.37 & 27.91 & 62.15 \\
\hline 65 & 1.48 & 18.75 & 2.76 & 30.05 & 3.56 & 62.60 & 3.65 & 6.95 & 27.25 & 66.15 \\
\hline 105 & 0.65 & 18.39 & 2.20 & 29.95 & 3.13 & 70.86 & 9.32 & 20.80 & 28.05 & 67.65 \\
\hline 145 & 0.26 & 18.75 & 2.09 & 30.05 & 2.58 & 71.34 & 13.15 & 23.35 & 28.11 & 67.15 \\
\hline 190 & 0.61 & 18.85 & 2.87 & 29.95 & 3.69 & 64.44 & 14.36 & 24.62 & 28.09 & 67.05 \\
\hline 240 & 0.15 & 18.65 & 1.55 & 29.65 & 2.85 & 54.28 & 14.45 & 24.73 & 29.57 & 68.48 \\
\hline 280 & 0.35 & 18.21 & 1.25 & 29.45 & 3.62 & 45.99 & 14.27 & 25.48 & 29.81 & 69.65 \\
\hline 325 & 0.15 & 19.18 & 2.73 & 29.25 & 2.35 & 41.89 & 11.96 & 28.05 & 30.39 & 69.35 \\
\hline
\end{tabular}

Table 4. Absolute error in the estimated temperatures from empirical models for samples at $843 \mathrm{~K}$.

\begin{tabular}{|c|c|c|c|c|c|c|c|c|c|c|c|c|}
\hline \multirow{3}{*}{ Model } & \multirow{3}{*}{$\begin{array}{l}\text { Sa- } \\
\text { mple }\end{array}$} & \multicolumn{11}{|c|}{$\Delta T=\mid T_{\text {True }}-T_{\text {Estimated }}(\mathrm{K})$} \\
\hline & & \multicolumn{11}{|c|}{ Heating time (min) } \\
\hline & & 1 & 5 & 12 & 35 & 65 & 105 & 145 & 190 & 240 & 280 & 325 \\
\hline & 1 & 95.3 & 99.7 & 106 & 127 & 136 & 121 & 97 & 66.6 & 33 & 9.8 & 8 \\
\hline \multirow[t]{2}{*}{ EM1 } & 2 & 51.7 & 51.4 & 52.3 & 56 & 56.8 & 53.8 & 51 & 40.8 & 34.9 & 27.4 & 17.4 \\
\hline & 3 & 20.5 & 19.2 & 17.3 & 12.8 & 7.1 & 0.3 & 3.9 & 7.5 & 11.1 & 12.3 & 16.5 \\
\hline & 1 & 97 & 105 & 108 & 129 & 141 & 131 & 113 & 88.8 & 61.4 & 41.9 & 27.5 \\
\hline \multirow[t]{2}{*}{ EM2 } & 2 & 54.6 & 54.6 & 55.7 & 60 & 62.1 & 60.8 & 59.6 & 51.9 & 47.6 & 41.9 & 33.7 \\
\hline & 3 & 24.7 & 23.8 & 22.5 & 18.9 & 14.5 & 8.9 & 5.2 & 2.4 & 0.7 & 1.4 & 5.2 \\
\hline & 1 & 54.5 & 52.7 & 48.6 & 33.7 & 19.8 & 11.9 & 9.3 & 10.9 & 15.7 & 18.8 & 18.9 \\
\hline \multirow[t]{2}{*}{ EM3 } & 2 & 46.7 & 45.3 & 43.4 & 38.8 & 32.3 & 26.3 & 21.5 & 18.4 & 17.2 & 15.9 & 16.1 \\
\hline & 3 & 22.2 & 21.6 & 19.5 & 17.6 & 15.3 & 14.9 & 13.5 & 12.3 & 12.7 & 12.1 & 11.8 \\
\hline \multirow{3}{*}{ EM4 } & 1 & 95.3 & 99.7 & 106 & 127 & 136 & 121 & 96.9 & 66.6 & 32.9 & 9.8 & 8 \\
\hline & 2 & 51.7 & 51.4 & 52.3 & 55.9 & 56.8 & 53.8 & 50.9 & 40.8 & 34.9 & 27.3 & 17.4 \\
\hline & 3 & 20.5 & 19.2 & 17.3 & 12.8 & 7.1 & 0.3 & 3.9 & 7.5 & 11.1 & 12.3 & 16.5 \\
\hline \multirow{3}{*}{ EM5 } & 1 & 110 & 113 & 121 & 145 & 164 & 160 & 139 & 94.9 & 31.2 & 17.9 & 55.3 \\
\hline & 2 & 62.7 & 62.9 & 63.9 & 68.9 & 70.8 & 68.4 & 65.3 & 53.1 & 45.1 & 34.3 & 20.3 \\
\hline & 3 & 24.8 & 23.5 & 21.1 & 15.6 & 8.4 & 0.0 & 5.7 & 10.7 & 15.3 & 17.2 & 22.7 \\
\hline \multirow{3}{*}{ EM6 } & 1 & 25.6 & 27.7 & 32.3 & 49.1 & 64.8 & 73.9 & 78.5 & 77.1 & 73.3 & 69.8 & 69.8 \\
\hline & 2 & 29.7 & 31.5 & 33.4 & 39.6 & 47.1 & 53.9 & 58.9 & 61.9 & 62.9 & 64.2 & 63.4 \\
\hline & 3 & 41.1 & 42 & 44.1 & 45.4 & 47.1 & 46.8 & 46.2 & 46.8 & 45.8 & 47.2 & 45.3 \\
\hline \multirow{3}{*}{ EM7 } & 1 & 97.4 & 102 & 108 & 129 & 141 & 133 & 117 & 95.8 & 71.8 & 54.9 & 43.2 \\
\hline & 2 & 56.3 & 56.5 & 57.7 & 62.4 & 65.2 & 65.1 & 64.9 & 59.2 & 55.9 & 51.7 & 45.2 \\
\hline & 3 & 30.5 & 30 & 29.7 & 27.5 & 24.9 & 21 & 18.4 & 16.9 & 14.6 & 14.7 & 11.7 \\
\hline \multirow{3}{*}{ EM8 } & 1 & 6.6 & 8.8 & 13.9 & 31.8 & 48.1 & 56.9 & 59.9 & 56.5 & 50.5 & 45.4 & 43.9 \\
\hline & 2 & 4.2 & 5.8 & 7.7 & 13.7 & 20.8 & 27.2 & 31.9 & 34.5 & 35.3 & 36.1 & 35 \\
\hline & 3 & 14.7 & 15.4 & 17.4 & 18.8 & 20.5 & 20.3 & 20.3 & 20.9 & 20.1 & 21.1 & 20 \\
\hline \multirow{3}{*}{ EM9 } & 1 & 85.9 & 90.6 & 97.3 & 115 & 119 & 96.1 & 64.2 & 28.9 & 9.1 & 34.1 & 53.6 \\
\hline & 2 & 39.8 & 38.7 & 39.4 & 41.4 & 40.1 & 34.3 & 29.4 & 16.1 & 8.6 & 1 & 12.4 \\
\hline & 3 & 6.1 & 4.3 & 1.2 & 4.8 & 12.3 & 20.4 & 25.2 & 29.7 & 33.7 & 35.7 & 39.9 \\
\hline \multirow{3}{*}{ EM10 } & 1 & 827 & 840 & 874 & 981 & 1069 & 1087 & 1067 & 985 & 913 & 840 & 793 \\
\hline & 2 & 493 & 499 & 499 & 526 & 540 & 546 & 547 & 536 & 528 & 520 & 502 \\
\hline & 3 & 273 & 274 & 276 & 272 & 267 & 260 & 247 & 242 & 238 & 242 & 228 \\
\hline \multirow{3}{*}{ EM11 } & 1 & 11.5 & 18.7 & 23.2 & 27.5 & 6 & 76.6 & 145 & 199 & 252 & 281 & 303 \\
\hline & 2 & 50.6 & 56.2 & 57.4 & 65.5 & 80.4 & 102 & 119 & 148 & 164 & 183 & 201 \\
\hline & 3 & 98.2 & 104 & 114 & 128 & 145 & 161 & 168 & 177 & 184 & 190 & 194 \\
\hline
\end{tabular}


10.21611/qirt.2019.048

Table 5. Best fitted models for samples at $843 \mathrm{~K}$.

\begin{tabular}{|c|c|c|c|c|c|c|c|c|c|}
\hline \multirow{3}{*}{$\begin{array}{l}\text { Heating } \\
\text { time } \\
\text { (min) }\end{array}$} & \multicolumn{9}{|c|}{ True temperature $=843 \mathrm{~K}$} \\
\hline & \multicolumn{3}{|c|}{ Sample 1} & \multicolumn{3}{|c|}{ Sample 2} & \multicolumn{3}{|c|}{ Sample 3} \\
\hline & Model & $\begin{array}{l}\text { Std error } \\
\text { in } T(K)\end{array}$ & $\mathrm{R}^{2}$ & Model & $\begin{array}{l}\text { Std error } \\
\text { in } T(K)\end{array}$ & $\mathrm{R}^{2}$ & Model & $\begin{array}{l}\text { Std error } \\
\text { in } \mathrm{T}(\mathrm{K})\end{array}$ & $\mathrm{R} 2$ \\
\hline 1 & EM8 & 15.3 & 0.993 & EM8 & 7.9 & 0.997 & EM9 & 5.5 & 0.999 \\
\hline 5 & EM8 & 15.8 & 0.993 & EM8 & 7.5 & 0.997 & EM9 & 5.4 & 0.999 \\
\hline 12 & EM8 & 16.2 & 0.993 & EM8 & 7.3 & 0.997 & EM9 & 5.6 & 0.999 \\
\hline 35 & EM11 & 79.3 & 0.999 & HR & 5 & 0.999 & EM9 & 6.2 & 0.999 \\
\hline 65 & EM11 & 129.4 & 0.996 & HR & 4.5 & 0.999 & EM1,4 & 8.1 & 0.999 \\
\hline 105 & EM3 & 30.3 & 0.969 & HR & 6.2 & 0.999 & EM5 & 8.7 & 0.999 \\
\hline 145 & EM3 & 27.6 & 0.975 & HR & 6.7 & 0.999 & EM1,4 & 6.4 & 0.999 \\
\hline 190 & EM3 & 23.6 & 0.98 & HR & 9.7 & 0.997 & EM2 & 7.3 & 0.999 \\
\hline 240 & EM9 & 81.6 & 0.985 & EM9 & 32.6 & 0.996 & EM2 & 7.5 & 0.998 \\
\hline 280 & EM1,4 & 66.2 & 0.985 & EM9 & 32.9 & 0.996 & EM2 & 6.7 & 0.999 \\
\hline 325 & EM1 4 & 52.3 & 0.99 & $\mathrm{HR}$ & 16.11 & 0.991 & EM2 & 5.9 & 0.999 \\
\hline
\end{tabular}

